\title{
Logistics risk and demand research based on neural network prediction Xiaoli Wang
}

Zhengzhou university of industrial technology. Henan. China

Keywords: Logistics industry; Economic development; Warehouse building

\begin{abstract}
The logistics industry plays an important role in the economic development,almost every city logistics park has been established.Zhengzhou logistics industry is still in its infancy,but development is rapidly,effectively support the development of regional economic development and change of the pattern of economic development.Zhengzhou lack of understanding of modern logistics, most enterprises can not understand modern logistics, so the current has a lot of logistics park, distribution center internal land design is not reasonable,there is no scientific logistics facilities, there is no comprehensive warehouse building, there is no reasonable guidance, the backward logistics industry make land utilization of the underground, serious resource wasted.
\end{abstract}

\section{INTRODUCTION}

Hsu found that a three-layer BP neural network can solve random function's fitting and approximation problem. As a result, a three-layer BP neural network is adopted in this paper. As shown in Fig. 1, BP neural network has three layers: input layer, hidden layer and output layer.

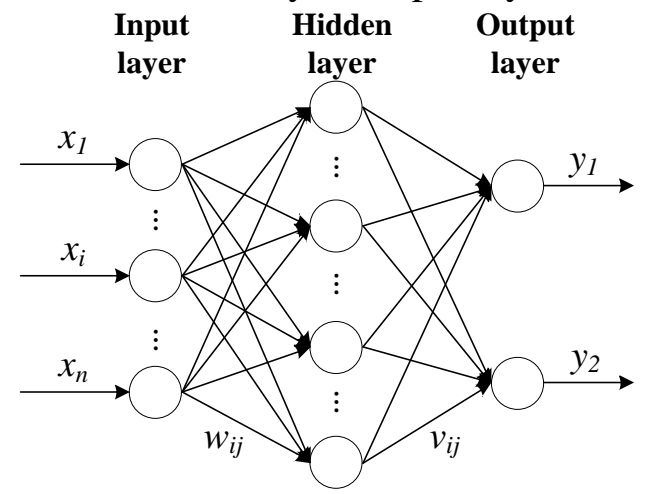

Fig. 1 BP neural network structure

Input variables are composed by the design variables $X\left(X=\left\{x_{1}, x_{2}, \cdots, x_{n}\right\}\right)$. The results of conflict detection can be divided into two kinds: one with conflicts and another without conflicts. The expression of $(0,1)$ represents the result without conflicts, while $(1,0)$ means conflicts occurred. So the number of hidden layer neurons nodes can be given by [16]:

$$
n_{2}=\sqrt{n_{1}+n_{3}}+\alpha
$$

Where $n_{1}$ is the number of input layer neurons nodes; $n_{3}$ is the number of output layer neurons nodes; $\alpha$ is a constant, produced random among $[0,1]$.

The dimensions of variables and goals are different in the design process. If parameters are used to detect conflict directly, the error precision of BP neural network will reduce. Before feeding the data into BP neural network, the data must be normalized in [0.1, 0.9], according to the following equation:

$$
x_{i}^{\prime}=0.1+\frac{x_{i}-x_{\min }}{x_{\max }-x_{\min }} *(0.9-0.1)
$$

Where $x_{i}$ is the input variables for all $i ; x_{\min }$ is the minimum of the data, while $x_{\max }$ is the maximum; $x_{i}$ is the input variables after normalization.

\section{Learning algorithm of BP neural network}

The mean square error of the actual and the target output is taken as the training error function of 
the BP neural network, defined as:

$$
E=\frac{1}{2 m q} \sum_{j=1}^{q} \sum_{i=1}^{m}\left(y_{i j}-z_{i j}\right)^{2}
$$

Where $m$ is the total number of samples; $q$ is the number of output layer neurons nodes; $y_{i j}$ is the actual output, while $z_{i j}$ is the target output.

To improve the convergence speed and generalization, additional momentum method and adaptive training algorithm are used to train the BP neural network [17]. Weight matrix can be amended as follows:

$$
w^{t+1}=w^{t}-\left.\eta \frac{\partial E}{\partial w}\right|_{w=w^{t}}+\left.a \eta \frac{\partial E}{\partial w}\right|_{w=w^{t+1}}
$$

Where $a$ is dynamic factor, produced random among [0,1]; $\eta$ is learning rate; $t$ is iterative steps; $w^{t}$ is the weight matrix, while $w^{t+1}$ is the weight matrix of next generation.

\section{Process of BP neural network optimized by immune algorithm}

For the sake of better initial weights and thresholds, IA is used to optimize the weights and thresholds of BP neural network. Fig. 2 shows a flow chart of BP neural network optimized by IA.

a) Antibody encoding

By using the binary encoding, individual is made up of connection weights between input layer and hidden layer, thresholds of hidden layer, connecting weights between hidden layer and output layer and thresholds of output layer. Table 1 shows the individual code of BP neural network. The number of antibodies consists of $n$ new antibodies and $m$ memory cells. Therefore, the total number of initial antibodies is $p$, the sum of $n$ and $m$.

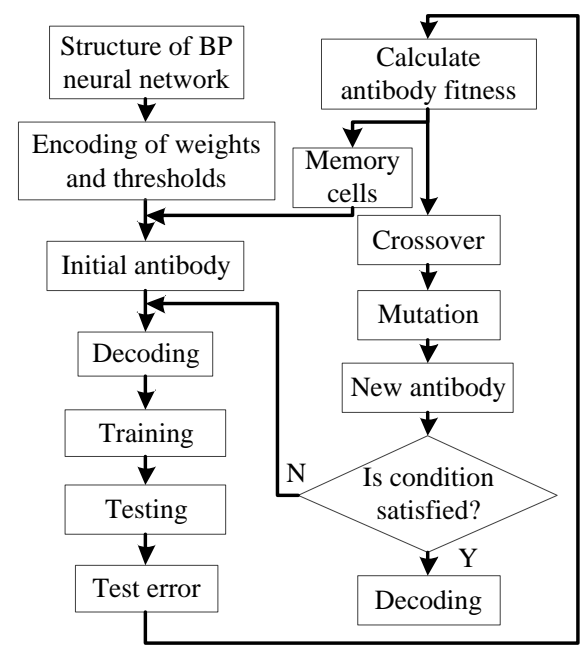

Fig. 2 Flowchart of BP network optimized by IA

Table 1 Antibody encoding

\begin{tabular}{cccc}
\hline $\begin{array}{c}\text { Connecting weights } \\
\text { between input layer } \\
\text { and hidden layer }\end{array}$ & $\begin{array}{c}\text { Thresholds } \\
\text { of hidden } \\
\text { layer }\end{array}$ & $\begin{array}{c}\text { Connecting weights } \\
\text { between hidden } \\
\text { layer } \\
\text { and output layer }\end{array}$ & $\begin{array}{c}\text { Thresholds } \\
\text { of output } \\
\text { layer }\end{array}$ \\
\hline$n_{1} \times n_{2}$ & $n_{2}$ & $n_{2} \times n_{3}$ & $n_{3}$ \\
\hline
\end{tabular}

b) Fitness function

For the purpose of less error, fitness function can be defined as:

$$
f\left(x_{i}\right)=\frac{1}{E\left(x_{i}\right)+\xi}
$$

Where $E\left(x_{i}\right)$ is mean square error of antigen; $\xi$ is penalty factor.

c) Concentration of antibody

To ensure the diversity of antibodies, a method based on the Euclidean distance is used to 
calculate the concentration of antibody. Concentration of the antibody calculated by:

$$
V\left(x_{i}\right)=\frac{1}{e^{D_{x_{i}}}}
$$

Where $D_{x_{i}}$ is the sum of all the Euclidean distances of antibodies. The higher the concentration of antibody is, the more similar between antibodies.

d) Expected reproduction probability

The expected reproduction probability is determined by the fitness $f\left(x_{i}\right)$ and concentration $V\left(x_{i}\right)$, which can be written by:

$$
p\left(x_{i}\right)=\lambda \frac{f\left(x_{i}\right)}{\sum f\left(x_{i}\right)}+(1-\lambda) \frac{V\left(x_{i}\right)}{\sum V\left(x_{i}\right)}
$$

Where $\lambda$ is the reproduction probability. From the above formula, we can conclude that when the concentration of antibody is high, the antibody with high fitness is hard to be selected; when the concentration of antibody is low, the antibody with high fitness is easy to be selected. By this way, good individual is withheld, the choice of similar antibody is reduced, and the variation of individual is ensured.

e) Genetic manipulation

The basic purpose of an immune system is to recognize foreign cells and molecules. If the difference between an antibody and an antigen is smaller, the affinity between an antibody and an antigen is higher, so recognition is also more likely.

1) Selection: stochastic uniform selecting method is used [18].

2) Crossover: one-point crossing method is used.

3) Mutation: uniform mutation is used for experimentation.

\section{Validation of IABP}

Designers have designed 14 schemes for the compound planetary gear train shown in Fig. 1, and the set of constraints $C_{1}$ have been verified. The teeth number and modulus of gears are fed into the BP neural network as input variables. Table 2 shows the normalized data according to Eq. (9), in which No. 1-10 are training samples and No. 11-14 are testing samples. Where $S_{1}$ and $p_{1}$ are the teeth numbers of sun gear and planetary gear in the first stage planetary gear train; $S_{2}$ and $p_{2}$ are the teeth numbers of sun gear and planetary gear in the second stage planetary gear train; $r$ is the teeth number of ring gear; $a$ and $b$ are the teeth numbers of the gear pair; $m_{1}$ and $m_{2}$ are the modulus of planetary gear train and parallel shaft.

\section{Conclusion}

The author thinks the logistics park promote the modernization as guide, it should be based on the existing, efforts to achieve three new progress.First, it is achieve new progress in scientific planning. According to the structure of large, medium and small three class, far, near the layout of the three levels, to formulate a scientific development plan adjustment. To prevent the logistics industry lack of land use types at the same time, also prevent redundant construction logistics industry land. Second, it is to achieve new progress on the key industrial park construction. From Zhengzhou the industry characteristics and the reality of the reality of logistics park construction demand, should highlight three key points: namely the expansion of the FAW logistics park project; Livestock products logistics park construction projects; Zero burden of logistics park planning project. Third, it is to achieve new progress on the logistics park upgrade into class. Based on the experience of the Shanghai, Beijing and other places to concentrate financial and material resources and energy, cultivate at least one modernization level higher park, guide the urban construction of modern logistics. This paper, by means of empirical study of Zhengzhou logistics industry made a careful investigation, land space for Zhengzhou logistics industry organization pattern research. 
Table 2 Data samples

\begin{tabular}{clllccccccc}
\hline No & \multicolumn{1}{c}{$S_{1}$} & \multicolumn{1}{c}{$p_{1}$} & \multicolumn{1}{c}{$S_{2}$} & \multicolumn{1}{c}{$p_{2}$} & \multicolumn{1}{c}{$r$} & \multicolumn{1}{c}{$a$} & \multicolumn{1}{c}{$b$} & $m_{1}$ & $m_{2}$ & Confli \\
. ct
\end{tabular}

\section{Reference}

[1] H. S. Zhao, L. Tian, B. S. Tong. Constraint-Based Conflict Detection and Negotiation in Collaborative Design. Computer Integrated Manufacturing Systems, Vol.8, No.11, 2002, pp.896-900.

[2] H. C. Xie, D. R. Chen, X. M. Kong. Constraint-based Conflict Detection in Collaborative Design. China Mechanical Engineering, Vol.13, No.18,2002, pp.1590-1592.

[3] D. Y. Wang, W. D. Jin. Conflict detection algorithm in collaborative design. Computer Applications, Vol.27, No.3, 2007, pp.650-652.

[4] Y. Xiong, W. H. Fan, G. L. Xiong. Research and Realization of Distributed Conflict Detection System. Computer Engineering, Vol.35, No.20, 2009, pp.23-27.

[5] L. Jaulin. Interval constraint propagation with application to bounded-error estimation. Automatica, Vol.36, No.10, 2000, pp.1547-1552. 